\title{
Solution to precision mixology challenge
}

\author{
Juris Meija ${ }^{1}$
}

(C) Crown copyright in right of Canada 2016

The winner of the precision mixology challenge (published in volume 408, issue 1) is:

Sami T. Tuomivaara, Complex Carbohydrate Research Center, University of Georgia, Athens, GA, USA

The award entitles the winner to select a Springer book of his choice up to a value of $€ 100,-$.

Our Congratulations!

\section{Solution}

Alice has a three-decimal-digit digital balance, which can weigh up to $100 \mathrm{~g}$ [1]. If she performs a single six-fold dilution of the stock solution with the mass fraction of cadmium $w_{0}=$ $3000 \mu \mathrm{g} / \mathrm{g}$, then the following equation can be written for the mass fraction of cadmium in the final solution, $w_{\mathrm{Cd}}$ :

$w_{C d}=w_{0} \frac{m_{\text {aliquot }}}{m_{\text {final }}}$

where $m_{\text {aliquot }} \approx m_{\text {final }} / 6$. Best results are always obtained when $m_{\text {final }}$ is as large as possible, i.e., $100 \mathrm{~g}$ in this case. The uncertainty of $w_{\mathrm{Cd}}$ is estimated via uncertainty propagation as follows:

$$
\begin{aligned}
& \frac{u^{2}\left(w_{C d}\right)}{w_{C d}^{2}}=\frac{u^{2}\left(m_{\text {aliquot }}\right)}{m_{\text {aliquot }}^{2}}+\frac{u^{2}\left(m_{\text {final }}\right)}{m_{\text {final }}^{2}} \\
& \frac{u^{2}\left(w_{C d}\right)}{w_{C d}^{2}}=\frac{0.002^{2}}{100.0^{2} / 6^{2}}+\frac{0.002^{2}}{100.0^{2}}=0.00012^{2}
\end{aligned}
$$

Juris Meija

juris.meija@nrc.ca

1 Measurement Science and Standards, National Research Council Canada, 1200 Montreal Road, Ottawa, ON K1A 0R6, Canada
If Alice performs serial dilution of the stock solution $n$-times, each time diluting the stock $k_{i}$ times where $i=1$, $2, \ldots, n$ and $k_{1} \times k_{2} \times \ldots \times k_{n}=K=6$, then the following equation can be written for the mass fraction of cadmium in the final solution, $w_{\mathrm{Cd}}$ :

$w_{C d}=w_{0} \frac{m_{1, \text { aliquot }}}{m_{1, \text { final }}} \times \frac{m_{2, \text { aliquot }}}{m_{2, \text { final }}} \times \ldots \times \frac{m_{n, \text { aliquot }}}{m_{n, \text { final }}}$

The relative uncertainty of $w_{\mathrm{Cd}}$ from Eq. 4 can be evaluated similar as it was done for Eq. 1 . For $n$-step dilution,

$\frac{u^{2}\left(w_{C d}\right)}{w_{C d}^{2}}=\sum_{i=1}^{n} \frac{u^{2}\left(m_{i, \text { aliquot }}\right)}{m_{i, \text { final }}^{2} / k_{i}^{2}}+\sum_{i=1}^{n} \frac{u^{2}\left(m_{i, \text { final }}\right)}{m_{i, \text { final }}^{2}}$

Note that $m_{i, \text { final }}=(100 \pm 0.002) \mathrm{g}$ and $m_{i, \text { aliquot }}=(100 \pm$ $0.002) / k_{i} \mathrm{~g}$. Given that all mass uncertainties are equal,

$\frac{u^{2}\left(w_{C d}\right)}{w_{C d}^{2}}=\frac{u^{2}(m)}{m_{\text {final }}^{2}} \sum_{i=1}^{n}\left(1+k_{i}^{2}\right) \propto \sum_{i=1}^{n}\left(1+k_{i}^{2}\right)$

The best results are those with lowest relative uncertainty of $w_{\mathrm{Cd}}$ and we can find out what values of $n$ and $k_{i}$ in Eq. 6 satisfy this requirement. In other words, we are seeking the minimum value for $\sum_{i=1}^{n}\left(1+k_{i}^{2}\right)$ as a function of $n$ and $k_{i}$ given the constrain $k_{1} \times k_{2} \times \ldots \times k_{n}=K$. This condition is met when all dilution factors are equal, which means $k_{i}=\sqrt[n]{K}$. It remains now to find the optimal number of steps for serial dilution in order to achieve the desired dilution factor and guarantee the lowest relative uncertainty of $w_{\mathrm{Cd}}$. This amounts to finding the minimum for function $\sum_{i=1}^{n}\left(1+\sqrt[n]{K^{2}}\right)=n+n \sqrt[n]{K^{2}}$, which occurs at $n=2.8 \approx 3$ for $K=6$. The minimum at $n \approx 3$ is also shown in Fig. 1.

The best strategy for Alice is to serially dilute the 3000 $\mu \mathrm{g} / \mathrm{g}$ cadmium solution three times, each time using a 


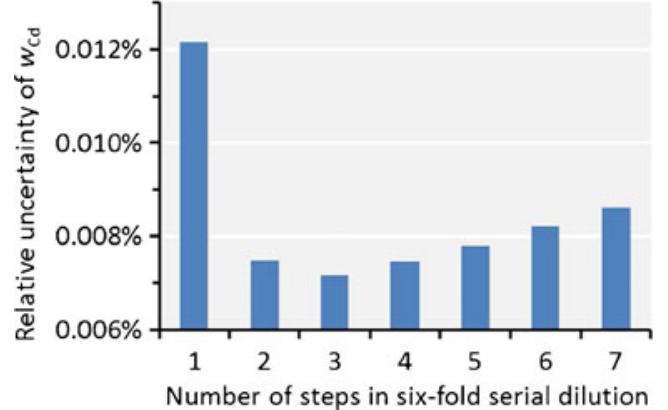

Fig. 1 Uncertainty of $w_{\mathrm{Cd}}$ when serial dilution of $3000 \mu \mathrm{g} / \mathrm{g}$ solution is performed in $n$ steps, each time with a dilution factor of $k_{i}=\sqrt[n]{6}(i=1,2$, $\ldots, n)$ dilution factor of $\sqrt[3]{6}$. In this problem we have looked only at the mathematical side of the problem and one has to acknowledge other sources of errors in dilutions. For example, while a three-step serial dilution might be preferable from a mathematical point of view, each step can lead to potential contaminations from glassware and pipettes.

\section{References}

1. Meija J. Anal Bioanal Chem. 2016;408:7. 